\title{
Research Paper: Clinimetrics of the Freezing of Gait Questionnaire for Parkinson Disease During the "off” State
}

\author{
Ghorban Taghizadeh $^{1} \mathbb{D}$, Seyed Mohammad Fereshtehnejad ${ }^{2,3} \mathbb{D}$, Pablo Martinez-Martin ${ }^{4}$, Mohammad Taghi Joghataei ${ }^{5,6}$ (D), Fatemeh \\ Mahdizadeh $^{7}$ (D), Sajad Sabbaghi ${ }^{8}$ (D), Sepide Goudarzi ${ }^{\text {(D), }}$, Mahsa Meimandi ${ }^{10}$ (D), Sayed Amir Hasan Habibi ${ }^{11}$ (D), Maryam Mehdizadeh ${ }^{5,6^{*}}$ (C) \\ 1. Department of Occupational Therapy, Rehabilitation Research Center, School of Rehabilitation Sciences, Iran University of Medical Science, \\ Tehran, Iran. \\ 2. Department of Neurobiology, Division of Clinical Geriatrics, Care Sciences and Society (NVS), Karolinska Institutet, Stockholm, Sweden \\ 3. Division of Neurology, Faculty of Medicine, University of Ottawa, Ottawa, ON, Canada. \\ 4. National Center of Epidemiology and CIBERNED, Carlos III Institute of Health, Madrid, Spain. \\ 5. Cellular and Molecular Research Center, Iran University of Medical Sciences, Tehran, Iran. \\ 6. Department of Neuroscience, School of Advance Technologies in Medicine, Iran University of Medical Sciences, Tehran, Iran. \\ 7. Neuromuscular Research Center, Semnan University of Medical Sciences, Semnan, Iran. \\ 8. Department of Occupational Therapy, School of Rehabilitation Sciences, University of Social Welfare Rehabilitation, Tehran, Iran. \\ 9. Department of Pharmacology and Toxicology, Faculty of Pharmacy, Tehran University of Medical Science, Tehran, Iran. \\ 10. Department of Occupational Therapy, School of Rehabilitation Sciences, Iran University of Medical Science, Tehran, Iran. \\ 11. Department of Neurology, Rasoul Akram Hospital, Iran University of Medical Science, Tehran, Iran.
}

\begin{tabular}{|l|l|}
$\begin{array}{l}\text { Use your device to san } \\
\text { and read the article online }\end{array}$ \\
Clinimetrics of the Freezing of Gait Questionnaire for Parkinson Disease During the “off”' State. Basic and Clinical Neuroscience, \\
12(1), 69-78. http://dx.doi.org/10.32598/bcn.12.1.882.11 \\
doi http://dx.doi.org/10.32598/bcn.12.1.882.11
\end{tabular}

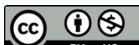

Article info:

Received: 23 Oct 2020

First Revision: 05 Nov 2020

Accepted: 22 Dec 2020

Available Online: 01 Jan 2021

Keywords:

Parkinson's disease, Gait, Questionnaire, Validity, Reliability, Diagnostic accuracy, “Off” state

\section{ABSTRACT}

Introduction: Freezing of gait, a common PD motor symptom, could increase the risk of falling. This study aimed to investigate the clinimetric attributes of the Freezing of Gait Questionnaire (FOGQ) for people with Parkinson disease in the "off”' state.

Methods: A total of 115 patients with Parkinson disease (PD; mean age, 60.25 years) were included. Acceptability, internal consistency (by the Cronbach alpha, and test-retest by Intraclass Correlation [ICC]), and reliability of the Persian-translated version of the FOGQ were examined. Dimensionality was estimated by Exploratory Factor Analysis (EFA). Fall efficacy scale-international, unified Parkinson disease rating scale-II, Berg balance scale, functional reach test, and Parkinson disease questionnaire-39 were applied to determine the convergent validity. Diagnostic accuracy for obtaining optimal cutoff point, separating faller and non-faller groups, was analyzed by Receiver Operating Characteristics (ROC) curve analysis and Area Under the Curve (AUC). All tests were carried out in an "off” state.

Results: The Cronbach alpha was high $(\alpha=0.92)$. The test-retest showed high reliability $(\mathrm{ICC}=0.89)$. The FOGQ was unidimensional according to the EFA and had acceptable convergent validity with moderate to high correlation with other clinical scales. The optimal cutoff point to discriminate fallers from non-fallers during the "off" state was 9/10, with an AUC of 0.92 .

Conclusion: Our results suggest that the FOGQ has appropriate reliability, validity, and discriminative ability for measuring FOG in patients with PD during the "off" state.

\footnotetext{
* Corresponding Author: 


\section{Highlights}

- The freezing of gait questionnaire is used to assess freezing during gait and turning in Parkinson disease.

- Most people with Parkinson disease report freezing of gait in the "off" state with more intensity.

- Validity and reliability for freezing of gait questionnaire in "off” state are essential and valuable.

\section{Plain Language Summary}

Freezing of gait (FOG) is a common condition in Parkinson disease patients, in which the patient is unable to initiate a move spontaneously. When the level of L-Dopa, a major drug in PD, has a drop, the patient experiences unwanted motor symptoms; the state is called the "off" state. Reliable tools to evaluate the FOG objectively are an essential step in planning more efficient treatment procedures. FOG questionnaire has been a valid tool to assess the freezing of gait in the "on" state in PD patients; however, having a valid tool for the "off" state is crucial for objective measures. Our results suggest that the FOG questionnaire has satisfactory reliability and validity in evaluating FOG in an "off" state in idiopathic PD.

\section{Introduction:}

reezing of Gait (FOG) is an episodic inability to start walking or turning, which usually takes several seconds. It seems that one's feet are stuck to the ground, and the patient cannot move any longer, which results in increasing the risk of falling and declining the quality of life of patients suffering from Parkinson Disease (PD) (Chee, Murphy, Danoudis, Georgiou-Karistianis \& Iansek, 2009; Plotnik, Giladi, Balash, Peretz \& Hausdorff, 2005). This phenomenon can sometimes be unpredictable and under the influence of psychological conditions (such as anxiety) (Ehgoetz Martens et al., 2016). According to former studies and evidence, most people with PD complain about FOG in the "off" state (when the medication effects are worn off) with more severity and frequency (more frequent and prolonged), while fewer people indicate these episodes in the "on" state (when the beneficial effect of the medication is present). Moreover, the duration of FOG in the "on" state is usually considerably shorter than in the "off" state (Nonnekes et al., 2015; Schaafsma et al., 2003).

It has been reported that levodopa may be beneficial in relieving FOG (though not constantly); however, it is hard to decide if a higher dose can result in a better effect (Schaafsma et al., 2003). Clinical symptoms vary from the "on" state to the "off" state, to the extent that FOG in the "off" state should be regarded as a different condition. According to experts, clinical evaluations conducted in an "off" state allow us to understand better the role of pharmacological and non-pharmacological treatments for FOG in patients with PD (Morris, 2000; Snijders et al., 2008). Therefore, a valid instrument to define and assess FOG (specifically in the "off" state) can play an important role not only in identifying this phenomenon but also for efficient medication and rehabilitation strategies (Nutt et al., 2011).

In 2000, Giladi et al. designed a 6-item questionnaire to quantify FOG (FOGQ). There are 6 items in the questionnaire, each with a score ranging from 0 to 4 , and a total score between 0 to 24 . The first two items focus on common difficulties with gait in PD patients. The last 4 items assess FOG frequency (item 3) and duration (items 4 to 6) (Giladi et al., 2000). The clinimetric properties of the FOG questionnaire have been assessed in several studies, but only in the "on" state (Oliveira Baggio, Curtarelli, Rodrigues \& Tumas, 2012; Candan, Çatıker \& Özcan, 2019; Giladi et al., 2009; Nilsson \& Hagell, 2009; Nilsson et al., 2010; Tambasco et al., 2015; Vogler, Janssens, Nyffeler, Bohlhalter \& Vanbellingen, 2015).

FOG occurs much more often in the "off" state-almost in $\% 90$ of the freezes. Additionally, the symptoms are usually more intense in the "off" state. However, dopaminergic medications may also cause FOG, though not often (Nonnekes et al., 2015; Snijders et al., 2008). Thus, in this study, we aimed to measure clinimetric attributes of the FOGQ in patients with PD in the "off" state. 


\section{Materials and Methods}

\subsection{Study Participants}

In this study, 115 patients with Parkinson disease (33 females and 82 males) with a Mean \pm SD age of 60.23 \pm 12.31 years were recruited from the outpatient clinics. The inclusion criteria were as follows: 1) clinical diagnosis of idiopathic Parkinson disease based on the United Kingdom Brain Bank criteria, 2) absence of major cognitive impairment (mini-mental status examination >21) (Azad et al, 2017); 3) absence of any concomitant impairment (orthopedic, neurologic, etc.) which can affect the functional state, and 4) ability to speak Persian and communicate fluently in this language. Subjects were excluded if they had a diagnosis of drug-induced Parkinsonism or if they use a drug that affects balance.

Ethical approval was granted by the Student Research Committee of the Iran University of Medical Science (Ethical Code: IR.IUMS.rec.1393.93-02-193-24848). Written informed consent was acquired from all participants before their inclusion in the study.

\subsection{Study procedure}

A trained therapist completed the FOGQ to make sure patients understood the concept of freezing of gait (the therapist explained or showed feet getting glued to the ground in various conditions). Notably, adequate timerest intervals were used between and within tests. To confirm the reliability, a 7-to-10 days' interval was considered between the test and retest. Other scales applied to construct the validity included Berg Balance Scale (BBS), Functional Reach (FR), unified Parkinson Disease Rating Scale-ADL (UPDRS), fall efficacy scale-international (FES-I), Parkinson Disease Questionnaire-39 (PDQ-39), and Schwab and England (S-E) activities of daily living scale, which the individuals completed during the first session. The tests were carried out in random order for each subject. All tests were conducted in the same room. The freezing of gait was assessed in an "off" state (12 hours after the last levodopa or anti-Parkinson dose) and an "on" state (1 hour after levodopa or antiParkinson dose) (Morris, 2000).

\subsection{Study instruments}

The Berg Balance Scale (BBS) assesses a person's performance based on 14 items associated with maintaining balance frequently used in daily life. The scoring of this measure is based on a 5-point ordinary scale ranges from 0 to 4 . This scale is a valid and reliable tool in Iranian patients with PD (Babaei-Ghazani et al., 2017).

Schwab and England (S-E) activities of daily living scale evaluate disability related to daily living activity in people with PD. The score ranges between 0 and 100, in which greater scores indicate a better functional state (Schwab, 1969).

The Parkinson Disease Questionnaire-39 (PDQ-39) is a 39-item, PD-specific, health-related quality-of-life measure that evaluates 8 different domains: mobility and Daily Living (ADL), emotional well-being, stigma, social support, cognition, communication, and body discomfort. The scoring of each item is based on a 5-point Likert-type scale ( 0 to 4$)$, in which a lower score indicates a better-perceived quality state (Giladi et al., 2000).

Fall Efficacy Scale-International (FES-I), as a valid and reliable tool in the Persian language, evaluates the fear of falling using 16 items while performing ADL. This measure is scored with a 5-point Likert-type scale (0-4), and a greater score represents higher fear of falling in ADL (Azad, Hassani Mehraban, Mehrpour \& Mohammadi, 2014).

Functional Reach Test (FRT) was designed to assess balance in older subjects based on the range reached as far forward as possible. It is found to have an excellent test-retest and inter-rater reliability in older people. Analysis of this measure is based on an average score of three trials. The complete description of this test is available in Newton's study (Newton, 2001).

Unified Parkinson Disease Rating Scale (UPDRS), a commonly used reference scale in PD studies, assesses different clinical aspects of PD (including non-motor aspects [part I], motor symptoms [part II], motor signs [part III], and motor complications [part IV]). In this study, we only used the ADL component of UPDRS. Each item in this scale is scored on a scale of 0 to 4 . The maximum possible total score of this 13 -item subscale is 52 points, and a greater score indicates lower performance. Also, a freezing item of UPDRS was used to assess FOG in this study (Giladi et al., 2000).

The Freezing of Gait Questionnaire (FOGQ) inquires the subject's experiences on 6 items related to FOG during the previous week. It consists of 6 items, each scored $0-4$, with a total score ranging from 0 to 24 . Higher scores indicate a more noticeable FOG. It requires 5-10 minutes to fill in the FOGQ (Giladi et al., 2000). 


\subsection{Translation process:}

After receiving consent from the corresponding author of the FOGQ (Giladi et al., 2000), translation and cross-cultural adaptation of the FOGQ into the Persian language was conducted based on guidelines approved by the international quality of life assessment project (Aaronson et al., 1992). In the first step, two experienced translators who were native Persian speakers individually translated the English version into Persian. None of them were familiar with FOGQ. In a meeting with the researchers involved in this study, the two translators discussed the discrepancies and then agreed upon a preliminary Persian version. The Persian version of the FOGQ was then translated back into English by two native English people who were blind to the original scale and unaware of the study. In the next step, the differences between the original and the translated versions were assessed. Finally, the English to Persian to English translated version was reviewed by Prof. Giladi. The Persian version was evaluated for cultural suitability by 4 neurologists and 14 therapists to determine the questionable items. The translated and culturally refined version of the FOG questionnaire was reviewed to check whether the questions were understandable enough to patients and necessary modifications were implemented. These modifications were reevaluated by the principal group of reviewers to provide the final version.

\subsection{Statistical analysis}

Descriptive statistics of the questionnaire scores and demographic data were conducted using mean, Standard Deviation (SD) for numeric and frequency percentage for categorical variables. The significance level for all statistical tests was less than 0.05 (Ghaffari, Akbarfahimi \& Rostami, 2020).

Floor and ceiling effects were determined based on the lowest (score 0) and highest (score 24) score obtained in the FOGQ. The floor/ceiling effect lower/higher than $15 \%$ was considered acceptable. The limits for the FOGQ total score skewness were considered from -1 to +1 (Hays, Anderson \& Revicki, 1993). The internal consistency of the FOGQ was calculated by inter-item correlation, corrected item-total correlation, and Cronbach alpha. The Cronbach alpha is considered adequate if it is 0.70 or higher, and the inter-item correlation is deemed adequate if 0.20 or higher (Piedmont, 2014).

The test-retest reliability of the sum score of the FOGQ was computed with the intra-class correlation coefficient (ICC, 1-way, random effect) statistics, where the ICC value of 0.70 or greater indicates acceptable reliability. The Standard Error Measurement (SEM) was calculated to determine measurement precision related to the testretest reliability (SDpooled $\sqrt{ }[1-\mathrm{ICC}])$. The acceptable SEM should be less than 1/2 SDpooled (Wyrwich et al., 2005).

\subsection{Exploratory Factor Analysis (EFA)}

The principal component method with varimax rotation was used to evaluate the validity and dimensionality of the Persian version of FOGQ. To determine the adequate sampling number, Kaiser-Meyer-Olkin (KMO) and Bartlett's test of sphericity were used. In the KMO, values greater than 0.7 and Bartlett's, $\mathrm{P}<0.05$ were regarded adequate. The number of factors was considered appropriate when eigenvalues were greater than 1 (Kaiser's criterion) (Gorsuch, 1997).

Convergent validity was assessed by calculating the Spearman correlation coefficient (r) between the total score of the FOGQ and the BBS, FRT, UPDRS (ADL, freezing item), PDQ-39, S-E, and FES-I scores. The correlation is interpreted as high if the coefficient value is 0.60 or higher, moderate if it ranges between 0.30-0.59, and weak if it is less than 0.30 (Fisk et al., 2005). To investigate discriminative validity, a Wilcoxon paired $t$ test was computed between the total score of FOGQ during "on" and "off" states (Nova, Perracini \& Ferraz, 2004).

Receiver operating characteristic (ROC) curve analysis was used to determine the optimal cutoff point between the groups who had a history of falling and those who not. Patient with at least one episode of PD-related-fall in the past 6 months was considered history positive. The optimal point is regarded as the score with the highest sensitivity and specificity values. Areas under ROC curves are described as follows: 0-0.49, 0.50-0.70, 0.700.89 , and $0.90-1$, indicating that the test performance for discrimination is worse, acceptable, good, and excellent, respectively. The highest Youden index (sensitivity + specificity -1) was considered as the optimal cutoff point to separate participants based on their history of falling (Bewick, Cheek \& Ball, 2004).

\section{Results}

Table 1 presents the demographic data of the study participants. The ceiling (2.60\%) and the floor (9.56\%) effects and the skewness $(0.32)$ of the total score of FOGQ were within acceptable limits. 
Table 1. Demographic characteristics of people with idiopathic Parkinson disease and descriptive statistics of FOGQ ( $\mathrm{n}=115)$

\begin{tabular}{|c|c|c|}
\hline \multicolumn{2}{|l|}{ Character } & Mean $\pm S D$ (range); No. (\%) \\
\hline \multicolumn{2}{|l|}{ Age (y) } & $60.2 \pm 12.3(23-73)$ \\
\hline & Male & $82(71.30)$ \\
\hline \multicolumn{3}{|l|}{ Sex } \\
\hline & Female & $33(28.69)$ \\
\hline \multicolumn{2}{|l|}{ Mini-mental status examination } & $23.2 \pm 3 .(21-30)$ \\
\hline \multicolumn{2}{|l|}{ Disease duration (y) } & $6.3 \pm 5.1(1-25)$ \\
\hline \multicolumn{2}{|l|}{ Hoehn and Yahr stage (off) } & $2.2 \pm 1.1(1-5)$ \\
\hline \multicolumn{2}{|l|}{ Levodopa equivalent dose $(\mathrm{mg} / \mathrm{d})$} & $758.8 \pm 286.8(100-2400)$ \\
\hline & Faller & $70(60.86)$ \\
\hline \multicolumn{3}{|l|}{ History of falling in the 6 past months } \\
\hline & Non-faller & 45 (39.13) \\
\hline
\end{tabular}

NEUROSCIENCE

The Cronbach alpha value was 0.92 , indicating high internal consistency. The inter-item correlation and item-total correlations were more than the desired value, which is within the adequate range (Table 2). The ICC for the total scores of the FOGQ was 0.89 , indicating satisfactory test-retest reliability, and the value of the SEM was 2.21 (SDpooled =6.68).
In the EFA, the one-factor solution explains $70.5 \%$ of the total variance (Eigenvalue, 4.23; $\mathrm{KMO}=0.85$, Bartlett's test of sphericity $\mathrm{P}$ value $<0.001$ ).

Table 3 summarizes the correlation coefficients between the FOGQ score and other measures to assess convergent validity. The FOGQ score was highly correlated with the freezing item of the UPDRS, UPDRS-II, and FES-I scores $(r=0.71-0.79)$. Our results show a moder-

Table 2. Inter-item correlation for FOGQ in people with idiopathic Parkinson disease $(n=115)$

\begin{tabular}{|c|c|c|c|c|c|c|c|c|c|c|}
\hline \multirow{2}{*}{\multicolumn{2}{|c|}{ Item of FOGQ }} & \multicolumn{6}{|c|}{ Inter-item Correlation } & \multirow{2}{*}{ Item Total Correlation } & \multirow{2}{*}{$\begin{array}{l}\text { The Cronbach Alpha if } \\
\text { Item Deleted }\end{array}$} & \multirow{2}{*}{$\begin{array}{c}\text { The Cronbach } \\
\text { Alphwa }\end{array}$} \\
\hline & & 1 & 2 & 3 & 4 & 5 & 6 & & & \\
\hline \multirow{7}{*}{ 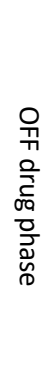 } & 1 & 1 & & & & & & 0.75 & 0.92 & \multirow{7}{*}{0.92} \\
\hline & 2 & 0.77 & 1 & & & & & 0.77 & 0.91 & \\
\hline & 3 & 0.55 & 0.60 & 1 & & & & 0.86 & 0.90 & \\
\hline & & & & & & & & & & \\
\hline & 4 & 0.47 & 0.51 & 0.74 & 1 & & & 0.84 & 0.90 & \\
\hline & 5 & 0.48 & 0.53 & 0.72 & 0.80 & 1 & & 0.87 & 0.89 & \\
\hline & 6 & 0.55 & 0.58 & 0.73 & 0.75 & 0.84 & 1 & 0.89 & 0.89 & \\
\hline
\end{tabular}

1. Walking during worst state;

2. Gait difficulties affecting daily living and independence;

3. Feet getting glued to the floor;
4. Duration of the longest freezing episode;

5. Duration of typical start hesitation;

6. Duration of typical turning hesitatio. 
Table 3. Correlation coefficients between total score of FOGQ with other scales in people with Idiopathic Parkinson disease ( $\mathrm{n}=115)$

\begin{tabular}{|c|c|c|c|c|c|c|c|c|}
\hline \multirow{2}{*}{ Scales } & \multirow{2}{*}{ BBS } & \multirow{2}{*}{ FRT } & \multirow{2}{*}{ UPDRS-II } & \multirow{2}{*}{$\begin{array}{c}\text { UPDRS } \\
\text { (Freezing Item) }\end{array}$} & \multicolumn{2}{|c|}{ PDQ-39 } & \multirow{2}{*}{ S-E } & \multirow{2}{*}{ FES-I } \\
\hline & & & & & Mobility & Total & & \\
\hline FOGQ & -0.64 & -0.53 & 0.79 & 0.71 & 0.70 & 0.58 & -0.64 & 0.77 \\
\hline
\end{tabular}

BBS: Berg Balance Scale; FRT: Functional Reach Test; UPDRS-II: Unified Parkinson Disease Rating Scale-II; SE: Schwab and England; PDQ-39: Parkinson Disease Questionnaire-39; FES-I: Fall Efficacy Scale-International; All test $<0.001$.

ate to a high correlation between balance measures (BBS and FR) with a total score of FOGQ $(|r=0.53-0.64|)$. The total score of the FOGQ was positively associated with the mobility subscale and the total score of the PDQ-39 and S-E $(|\mathrm{r}=0.58-0.70|)$.

A significant difference was observed between the total score of FOGQ in the "on" and" off" states (7.4 \pm 5.9 (Mean $\pm \mathrm{SD}$ ) in “on" vs. $9.8 \pm 6.9$ in "off” state; $\mathrm{P}<0.001$ ).

The optimal cutoff point was 9/10 to distinguish PD patients with and without a history of falling in the past 6 months. Sensitivity (95\% confidence interval) and specificity ( $95 \%$ confidence interval) values were 88.90 (75.90-96.30) and 92.86 (84.1-97.6), respectively. The AUC and Youden index were respectively 0.92 and 0.82 , using a cutoff point estimated at 9/10.

\section{Discussion}

The results of our study demonstrated that the FOGQ completed in an "off" state has satisfactory validity, high reliability, and high discriminant ability to differentiate PD patients with a history of falling from those without falling.

The ceiling and floor effects, as well as the skewness of the total score of FOGQ, verify the acceptability of data, which is consistent with earlier studies during "on" state (Nilsson \& Hagell, 2009; Nilsson et al., 2010; Tambasco et al., 2015).

FOGQ's internal consistency, in our study during “off” state, was excellent, which is in line with all previous FOGQ studies in "on" state (Oliveira Baggio et al., 2012; Giladi et al., 2000; Giladi et al., 2009; Nilsson \& Hagell, 2009; Nilsson et al., 2010; Vogler et al., 2015). This indicates a good and coherent association between the 6 items of the FOGQ. Test-retest reliability analysis was used to assess the stability of the measure during different times, and it was noted that the total score of FOGQ in the "off" state remains steady over time. Such reliability values are consistent with former reports exe- cuted only in the "on" state (Oliveira Baggio et al., 2012; Candan et al., 2019; Giladi et al., 2009; Nilsson et al., 2010). The low SEM in our study, which is similar to the results in the "on" state (Nilsson et al., 2010), approves the correctness of our measurement in the "off" state.

Investigation of dimensionality is a necessary element of construct validity, which can be done by EFA (Slocum-Gori \& Zumbo, 2011). Results of the EFA demonstrated that the FOGQ is a unidimensional assessment tool, indicating that various items of the FOGQ measure only one thing (i.e. FOG).

Regarding convergent validity, the FOGQ showed a high correlation with the UPDRS (freezing item) since both measures assess the same construct. Similar correlation coefficient values were found in previous studies in the "on" state (Oliveira Baggio et al., 2012; Giladi et al., 2000; Giladi et al., 2009; Nilsson \& Hagell, 2009; Nilsson et al., 2010; Tambasco et al., 2015). A moderate association was found between the FOGQ and balance measures (BBS and FR). This result indicates that in patients with PD, one of the causes of balance difficulties can be FOG, which is related to findings in the Brazilian and Turkish versions of the FOGQ in the "on" state (Oliveira Baggio et al., 2012; Candan et al., 2019). A high correlation was found between the FOGQ and two measures of activities of daily life, UPDRS-DL, and S-E ADL, which indicates the importance of freezing of gait in functional skills. Similar relationships have been previously reported in the "on" state (Oliveira Baggio et al., Giladi et al., 2000; Nilsson \& Hagell, 2009; Tambasco et al., 2015; Vogler et al., 2015). A moderate-to-high correlation was also found between the FOGQ and mobility subscale and the total score of the PDQ-39. This result confirms that the freezing of gait deteriorates the quality of life, and it was in accordance with the results from former studies in the "on" state (Delgado-Alvarado et al., 2020; Ellis et al., 2011; Giladi et al., 2000; Silva-Batista et al., 2020; Vogler et al., 2015). Furthermore, we found a high correlation between FOGQ and FES-I, indicating the association between FOG and fear of falling in PD. 
We also showed that the FOG questionnaire is a valid tool to discriminate "on" and "off" states, which would be valuable in clinical settings and research.

The FOGQ score in the "off" state can identify PD patients with a history of falling (in the past 6 months). Most of the studies considering PD-associated-falling have concluded that FOG could be one of its main causes. The progression of the disease can be one of the causes of further FOG and falling in people with Parkinson disease, but some studies have shown that FOG can occur in earlier stages of the disease (Bohnen et al., 2019; Giladi et al., 2001; Nonnekes et al., 2015). It can be helpful in future studies and therapeutic planning and also in the prediction of falling, markedly in an "off" state, during which the patient's condition worsens.

We acknowledge our study limitations, including a non-randomized sampling method and exclusion of patients with major cognitive impairment (which are indeed more prone to FOG). These limitations might affect the generalizability of our findings and need to be re-addressed in future studies. Yet, our study is one of the few to assess the clinimetric properties of the FOGQ during the "off" state.

In conclusion, the Persian version of the FOGQ is found to have a high test-retest reliability and internal consistency, and acceptable validity and accuracy in assessing freezing of gait during the "off' state and identifying patients with a history of falling.

\section{Ethical Considerations}

\section{Compliance with ethical guidelines}

This study was approved by the Ethics Committee of the Iran University of Medical Science (Ethical Code: IR.IUMS.rec.1393.93-02-193-24848).

\section{Funding}

This research did not receive any grant from funding agencies in the public, commercial, or non-profit sectors.

\section{Authors' contributions}

Conceptualization: Ghorban Taghizadeh, Sayed Amir Hasan Habibi, Maryam Mehdizadeh; Methodology: Seyed-Mohammad Fereshtehnejad, Pablo MartinezMartin, Ghorban Taghizadeh, and Sayed Amir Hasan Habibi; Formal analysis: Ghorban Taghizadeh, , Pablo Martinez-Martin, and Maryam Mehdizadeh; Investigation and data curation: Fatemeh Mahdizadeh, Sajad
Sabbaghi, Sepide Goudarzi, Mahsa Meimandi. Writing the original draft preparation: Ghorban Taghizadeh, Mohammad Taghi Joghataei, and Maryam Mehdizadeh; Writing, review, and editing: Ghorban Taghizadeh, Seyed-Mohammad Fereshtehnejad, Pablo MartinezMartin, Sepide Goudarzi, and Maryam Mehdizadeh.

\section{Conflict of interest}

The authors declared no conflict of interest.

\section{Acknowledgments}

We thank the patients with PD who kindly agreed to participate in this study. The study was supported by Mr. Taa'ti and Ms. Aghaiepour, which is also gratefully acknowledged.

\section{References}

Aaronson, N. K., Acquadro, C., Alonso, J., Apolone, G., Bucquet, D., \& Bullinger, M., et al. (1992). International quality of life assessment (IQOLA) project. Quality of Life Research, 1(5), 34951. [DOI:10.1007/BF00434949] [PMID]

Azad, A., Hassani Mehraban, A., Mehrpour, M., \& Mohammadi, B. (2014). Clinical assessment of fear of falling after stroke: Validity, reliability and responsiveness of the Persian version of the Fall Efficacy Scale-International. Medical Journal of the Islamic Republic of Iran, 28, 131. [PMID] [PMCID]

Azad, A., Taghizadeh, G., Ghorbanpoor, H., Lajevardi, L., \& Farhadian, M. (2017). Relationship between laterality and handedness with the higher order sensory functions and manual dexterity of the elderly. Iranian Rehabilitation Journal, 15(4), 367-76. [DOI:10.29252/nrip.irj.15.4.367]

Babaei-Ghazani, A., Mohammadi, H., Shahidi, Gh. A., Habibi, S. A. H., Forogh, B., \& Ahadi, T., et al. (2017). Reliability and validity of the Persian translation of Berg Balance Scale in Parkinson disease. Aging Clinical and Experimental Research, 29(5), 857-62. [DOI:10.1007/s40520-016-0682-7] [PMID]

Bewick, V., Cheek, L., \& Ball, J. (2004). Statistics review 13: Receiver operating characteristic curves. Critical Care, 8(6), 508. [DOI:10.1186/cc3000] [PMID] [PMCID]

Bohnen, N. I., Kanel, P., Zhou, Z., Koeppe, R. A., Frey, K. A., \& Dauer, W. T., et al. (2019). Cholinergic system changes of falls and freezing of gait in Parkinson's disease. Annals of Neurology, 85(4), 538-49. [DOI:10.1002/ana.25430] [PMID] [PMCID]

Candan, S. A., Çatıker, A., \& Özcan, T. Ş. (2019). Psychometric properties of the Turkish version of the freezing of gait questionnaire for patients with Parkinson's disease. Neurological Sciences and Neurophysiology, 36(1), 44-50. https://www.researchgate.net/publication/331935552

Chee, R., Murphy, A., Danoudis, M., Georgiou-Karistianis, N., \& Iansek, R. (2009). Gait freezing in Parkinson's disease and the 
stride length sequence effect interaction. Brain, 132(8), 215160. [DOI:10.1093/brain/awp053] [PMID]

Delgado-Alvarado, M., Marano, M., Santurtún, A., Urtiaga-Gallano, A., Tordesillas-Gutierrez, D., \& Infante, J. (2020). Nonpharmacological, nonsurgical treatments for freezing of gait in Parkinson's disease: A systematic review. Movement Disorders, 35(2), 204-14. [DOI:10.1002/mds.27913] [PMID]

Ehgoetz Martens, K. A., Hall, J. M., Gilat, M., Georgiades, M. J., Walton, C. C., \& Lewis, S. J. G. (2016). Anxiety is associated with freezing of gait and attentional set-shifting in Parkinson's disease: A new perspective for early intervention. Gait E Posture, 49, 431-6. [DOI:10.1016/j.gaitpost.2016.07.182] [PMID]

Ellis, T., Cavanaugh, J. T., Earhart, G. M., Ford, M. P., Foreman, K. B., \& Dibble, L. E. (2011). Which measures of physical function and motor impairment best predict quality of life in Parkinson's disease? Parkinsonism \& Related Disorders, 17(9), 693 7. [DOI:10.1016/j.parkreldis.2011.07.004] [PMID] [PMCID]

Fisk, J. D., Brown, M. G., Sketris, I. S., Metz, L. M., Murray, T. J., \& Stadnyk, K. J. (2005). A comparison of health utility measures for the evaluation of multiple sclerosis treatments. Journal of Neurology, Neurosurgery \& Psychiatry, 76(1), 58-63. [DOI:10.1136/jnnp.2003.017897] [PMID] [PMCID]

Ghaffari, A., Akbarfahimi, M., \& Rostami, H. R. (2020). Discriminative factors for post-stroke depression. Asian Journal of Psychiatry, 48, 101863. [DOI:10.1016/j.ajp.2019.101863] [PMID]

Giladi, N., Shabtai, H., Simon, E. S., Biran, S., Tal, J., \& Korczyn, A. D. (2000). Construction of freezing of gait questionnaire for patients with Parkinsonism. Parkinsonism \& Related Disorders, 6(3), 165-70. [DOI:10.1016/S1353-8020(99)00062-0]

Giladi, N., Tal, J., Azulay, T., Rascol, O., Brooks, D. J., \& Melamed, E., et al. (2009). Validation of the freezing of gait questionnaire in patients with Parkinson's disease. Movement Disorders, 24(5), 655-61. [DOI:10.1002/mds.21745] [PMID]

Giladi, N., Treves, T. A., Simon, E. S., Shabtai, H., Orlov, Y., \& Kandinov, B., et al. (2001). Freezing of gait in patients with advanced Parkinson's disease. Journal of Neural Transmission, 108(1), 53-61. [DOI:10.1007/s007020170096] [PMID]

Godefroy, O., Fickl, A., Roussel, M., Auribault, C., Bugnicourt, J. M., \& Lamy, C., et al. (2011). Is the Montreal Cognitive Assessment superior to the Mini-Mental State Examination to detect poststroke cognitive impairment? A study with neuropsychological evaluation. Stroke, 42(6), 1712-6. [DOI:10.1161/ STROKEAHA.110.606277] [PMID]

Gorsuch, R. L. (1997). Exploratory factor analysis: Its role in item analysis. Journal of Personality Assessment, 68(3), 532-60. [DOI:10.1207/s15327752jpa6803_5] [PMID]

Hays, R. D., Anderson, R., \& Revicki, D. (1993). Psychometric considerations in evaluating health-related quality of life measures. Quality of Life Research, 2(6), 441-9. [DOI:10.1007/ BF00422218] [PMID]

Morris, M. E. (2000). Movement disorders in people with Parkinson disease: A model for physical therapy. Physical Therapy, 80(6), 578-97. [DOI:10.1093/ptj/80.6.578] [PMID]

Newton, R. A. (2001). Validity of the multi-directional reach test: A practical measure for limits of stability in older adults. The Journals of Gerontology: Series A, 56(4), M248-52. [DOI:10.1093/ gerona/56.4.M248] [PMID]
Nilsson, M. H., \& Hagell, P. (2009). Freezing of Gait Questionnaire: Validity and reliability of the Swedish version. Acto Neurologica Scandinavica, 120(5), 331-4. [DOI:10.1111/j.16000404.2009.01175.x] [PMID]

Nilsson, M. H., Hariz, G. M., Wictorin, K., Miller, M., Forsgren, L., \& Hagell, P. (2010). Development and testing of a self administered version of the Freezing of Gait Questionnaire. BMC Neurology, 10, 85. [DOI:10.1186/1471-2377-10-85] [PMID] [PMCID]

Nonnekes, J., Snijders, A. H., Nutt, J. G., Deuschl, G., Giladi, N., \& Bloem, B. R. (2015). Freezing of gait: A practical approach to management. The Lancet Neurology, 14(7), 768-78. [DOI:10.1016/S1474-4422(15)00041-1]

Nova, I. C., Perracini, M. R., \& Ferraz, H. B. (2004). Levodopa effect upon functional balance of Parkinson's disease patients. Parkinsonism \& Related Disorders, 10(7), 411-5. [DOI:10.1016/j. parkreldis.2004.04.004] [PMID]

Nutt, J. G., Bloem, B. R., Giladi, N., Hallett, M., Horak, F. B., \& Nieuwboer, A. (2011). Freezing of gait: Moving forward on a mysterious clinical phenomenon. The Lancet Neurology, 10(8), 734-44. [DOI:10.1016/S1474-4422(11)70143-0]

Oliveira Baggio, J. A., Curtarelli, M. B., Rodrigues, G. R., \& Tumas, V. (2012). Validity of the Brazilian version of the freezing of gait questionnaire. Arquivos de Neuro-Psiquiatria, 70(8), 599603. [DOI:10.1590/S0004-282X2012000800008] [PMID]

Piedmont, R. L. (2014). Inter-item correlations. In A.C. Michalos (Eds.), Encyclopedia of quality of life and well-being research (pp. 3303-3304). Dordrecht: Springer. [DOI:10.1007/978-94-0070753-5_1493]

Plotnik, M., Giladi, N., Balash, Y., Peretz, C., \& Hausdorff, J. M. (2005). Is freezing of gait in Parkinson's disease related to asymmetric motor function? Annals of Neurology, 57(5), 65663. [DOI:10.1002/ana.20452] [PMID]

Schaafsma, J. D., Balash, Y., Gurevich, T., Bartels, A. L., Hausdorff, J. M., \& Giladi, N. (2003). Characterization of freezing of gait subtypes and the response of each to levodopa in Parkinson's disease. European Journal of Neurology, 10(4), 391-8. [DOI:10.1046/j.1468-1331.2003.00611.x] [PMID]

Schwab, R. S. (1969). Projection technique for evaluating surgery in Parkinson's disease. In F. J. Gillingham, I. M. L. Donaldson, \& Royal College of Surgeons of Edinburgh. Third symposium on Parkinson's disease (pp. 152-157). Edinburgh: E \& S Livingstone. https://ci.nii.ac.jp/naid/10005054625/

Silva-Batista, C., de Lima-Pardini, A. C., Nucci, M. P., Coelho, D. B., Batista, A., \& Pimentel Piemonte, M. E., et al. (2020). A randomized, controlled trial of exercise for Parkinsonian individuals with freezing of gait. Movement Disorders, 35(9), 1607-17. [DOI:10.1002/mds.28128] [PMID] [PMCID]

Slocum-Gori, S. L., \& Zumbo, B. D. (2011). Assessing the unidimensionality of psychological scales: Using multiple criteria from factor analysis. Social Indicators Research, 102(3), 443-61. [DOI:10.1007/s11205-010-9682-8]

Snijders, A. H., Nijkrake, M. J., Bakker, M., Munneke, M. Wind, C., \& Bloem, B. R. (2008). Clinimetrics of freezing of gait. Movement Disorders, 23(S2), S468-74. [DOI:10.1002/ mds.22144] [PMID]

Tambasco, N., Simoni, S., Eusebi, P., Ripandelli, F., Brahimi, E., \& Sacchini, E., et al. (2015). The validation of an Italian ver- 
sion of the Freezing of Gait Questionnaire. Neurological Sciences, 36(5), 759-64. [DOI:10.1007/s10072-014-2037-5] [PMID]

Vogler, A., Janssens, J., Nyffeler, T., Bohlhalter, S., \& Vanbellingen, T. (2015). German translation and validation of the "freezing of gait questionnaire" in patients with Parkinson's disease. Parkinson's Disease, 2015, 982058. [DOI:10.1155/2015/982058] [PMID] [PMCID]

Wyrwich, K. W., Bullinger, M., Aaronson, N., Hays, R. D., Patrick, D. L., \& Symonds, T. (2005). Estimating clinically significant differences in quality of life outcomes. Quality of Life Research, 14(2), 285-95. [DOI:10.1007/s11136-004-0705-2] [PMID] 
This Page Intentionally Left Blank 